\title{
A Feasibility Study Comparing Two Commercial TIG Welding Machines for Deep Penetration
}

\author{
Richard French $^{1}$, Hector Merin-Reyes ${ }^{1}$, and Will Yeadon ${ }^{1, *}$ \\ ${ }^{1}$ Department of Physics and Astronomy, The University of Sheffield, Sheffield, United Kingdom
}

\begin{abstract}
Developing a deep penetration TIG welding technique to produce welds of equal quality to the industrial standard practise of laser-based welding techniques has the potential to lower production complexity and cost. Higher currents levels are required to increase penetration depth in conventional TIG welding but this results in excessive weld bead width amongst other detrimental effects. However, through K-TIG and A-TIG techniques these detrimental effects can be circumnavigated. Prior experimental work on weld pool dynamics in conventional TIG welding in higher current regions has been sparse as TIG welding enhanced through novel techniques provides the best quality welds. This paper is an early feasibility study for novel deep penetration welding techniques motivated by observations made during research done at The University of Sheffield where unexpected activity in the weld pool was identified during TIG welding with a VBC IE500DHC between 300A - 1000A. This current range is labelled the 'Red Region'. Understanding the fluid dynamics of the molten metal in the weld pool at the 'Red Region' current level will help in the creation of novel techniques for deep penetration TIG welding. Addressing this, this paper compares the quality of welds produced between $100 \mathrm{~A}$ and $200 \mathrm{~A}$ on 316 Stainless Steel by two industrially leading welding machines; the Miller Dynasty 350 and the VBCie 500DHC.
\end{abstract}

\section{Introduction}

Achieving consistent high quality deep penetration welds is a difficult but potentially highly profitable endeavour. Due to complex activity in the weld pool, deep penetration welding requires the use of specialised techniques to push the weld pool deeper into the work piece. The prevailing industrial solutions of laser-based deep penetration techniques are expensive and cumbersome to transport. An off-the-shelf deeppenetration TIG technique may drastically reduce costs and expand the applications of deep penetration welding. TIG welding often does not require considerable preparation of the work piece and through novel modification of TIG welding, a technique which manipulates weld pool dynamics to achieve deep penetration could be developed.

TIG welding solutions for deep penetration require novel techniques to avoid the adverse effects of the large current required for deep penetration such as the bead width becoming excessively large. At present, the two most successful solutions are A-TIG and K-TIG. The Active flux TIG, or A-TIG, technique is a good demonstration of how the Marangoni effect can be manipulated to achieve deep weld penetration. A-TIG involves the addition of surfactants to the work piece to increase the surface tension in centre of the weld pool so that Marangoni convection pushes the weld pool deeper into the work piece. K-TIG, or Keyhole TIG, is an industrially popular TIG technique. The novel principle of K-TIG is to cool the weld torch by circulating water around it - this changes the shape of the arc such that a keyhole can be formed during welding.

Research at The University of Sheffield into deep penetration welding found $300 \mathrm{~A}$ to be a threshold current during TIG welding. From observations with a thermal camera it was observed that at and above 300A severe fluctuations in the weld pool began. This observation motivated this study into the possible causes of this activity and whether this effect could be used in the creation of a novel deep penetration technique. The current region this effect was prominent in, 300A $1000 \mathrm{~A}$, has been labelled as the 'Red Region'.

After reviewing the literature for past standard TIG welding research at this current range, no detailed documentation of prior observations of this effect were found [1-2]. A study by Lin and Eagar performed in 1984 looking at the influence arc pressure has on the geometry of the weld pool found 300 A to be the current at which the surface tension and hydrostatic potential energy is overcome by the arc pressure [3]. The subsequent deep depression in the weld pool could not completely be modelled just by considering the arc pressure but could be explained with the inclusion of a compound vortex - a forced vortex in the centre of the liquid weld pool surrounded by a free vortex. In addition to this, previous K-TIG studies have found $300 \mathrm{~A}$ to be the threshold for the surface tension to be overcome by the arc pressure [4]. However, this is with the increased energy densities achieved with the K-TIG technique so 
the behaviour of the weld pool under standard TIG welding may not be identical. This is in addition to the several input variables which determine the behaviour of the weld pool during welding and the quality of the weld produced. Factors such as the welding speed, arc gap and the material being welded may mask the physical mechanism that results in the effects observed in the 'Red Region'. Thus, to investigate the 'Red Region' an understanding of which factors are not involved is imperative.

To address this, this paper will briefly describe the factors affecting weld pool dynamics and TIG welding arc physics before reviewing prior deep penetration welding techniques. Following this, the experimental procedure of the undertaken work will be described and then the results will be presented and discussed.

\subsection{Weld Pool Dynamics}

The weld pool is highly active during welding with several competing effects resulting in complex motion of the molten metal. The main forces involved are the Marangoni effect, the Lorentz force and the Arc Pressure [5]. The Marangoni effect occurs during welding as there is a gradient in surface tension in the weld pool and metals have a variation of surface tension with temperature. So, molten metal in areas of lower surface tension flows towards areas of higher surface tension. For deep penetration, it is far better to have the centre of the weld pool at the highest surface tension - a positive gradient - as this results in Marangoni convections radially inwards. Small differences in the concentrations of surface active elements such as oxygen and sulphur can cause changes in the surface tension to temperature gradient in a weld pool and thus have a large effect on the weld penetration.

The Lorentz Force during welding is an artefact of the interaction between the current flow in the weld pool and the magnetic field it induces. This force is proportional to the arc current squared and so is stronger at higher currents [6]. In addition to this, electrical resistivity in a metal is temperature dependent with cooler temperatures resulting in less resistance. In arc welding, the concentration of heat reduces as distance from the centre of the electric arc increases. As current flow favours coolers areas of less resistance, the hotter central region of the weld pool will become increasingly inefficient at conducting current reducing the current density. The difference in current densities created by the differences in resistance results in the magnetic field created by the current pushing the weld pool downwards. The force is weaker near the bottom of the weld pool and this creates a toroidal vertex which results in significant heat transfer deeper into the weld pool.

Arc Pressure is caused by the incompressibility of the welding arc plasma. Thus the momentum the arc transfers when it impacts the weld pool pushes the surface downwards. An increase in arc pressure can cause the surface of the weld pool to become more concave [7]. In addition to the downward pressure from the welding Arc, the shield gas also acts to perturb the weld pool but with considerably lower pressure [8].

The force resulting from the Marangoni effect is the dominant force in weld pool dynamics, yet it is important to emphasise that it is not overwhelmingly dominant and in fact the electromagnetic force is particularly important at higher current (and thus temperature) levels. Additional forces such as buoyancy - from the different densities between the hotter and colder areas of the weld pool also affect weld pool dynamics but at an order of magnitude less than the Marangoni effect [5].

\subsection{TIG Welding Arc Physics}

TIG welding arcs are complex phenomena that are difficult to model accurately. Typically, electrons flow out of the electrode (cathode) and into the workpiece (anode) ionizing the shield gas to create a plasma which is mixture of approximately equal parts electrons and ionized shielding gas ions [9]. The electrons are emitted from the electrode through thermionic, thermo-field and field emission in addition to secondary emissions such as when highly energetic ions from the shielding gas collide with the electrode as well as electron emissions from the anode [10].

The properties of the arc, particularly anode current density and heat flux to the workpiece which determine the heat flow into the weld pool [11], strongly affect the quality of the weld produced. The Lorentz Force causes a 'pinching' of the arc increasing the energy density and the Arc Pressure from the momentum of the impinging arc plasma distorts the surface of the weld pool. The flow of the cathode plasma jet along the surface of the weld pool leads to an aerodynamic drag force that pushes the surface of the weld pool radially outwards. These various effects have different reliance on the arc current thus modelling of TIG welding arcs is complex and several approximations are typically made [12-14]. Usually, local thermodynamic equilibrium, LTE, and a flat weld pool surface is assumed. However, high currents produce large arc pressures - resulting in concave weld pool surfaces - and welding arcs are not in LTE [2].

\subsection{Deep Penetration Welding}

To achieve deep penetration welding with TIG the heat from the welding arc must penetrate far into the work piece creating a deep weld pool. The two prominent TIG based techniques that achieve this are Keyhole TIG and Activated Flux TIG. 
Keyhole TIG, or K-TIG, involves a small 'keyhole' punched through the work piece enabling the formation of a narrow cavity during welding which allows the heat from the welding arc to be distributed deep into the work piece. This keyhole remains open due to electromagnetically driven arc jets [15].

Standard TIG welding has an energy density of the order $10^{7} \mathrm{Wm}^{-2}[16]$. Typically, welding processes involving energy densities of between $10^{10}$ and $10^{13} \mathrm{Wm}^{-}$ 2 are required to achieve keyhole formation and stability using the recoil pressure - from the vaporising metal - to keep the keyhole open during welding [17]. Increasing energy densities further, to above $10^{13} \mathrm{Wm}^{-2}$, results in the work piece vaporising faster than the energy can be dissipated through it, thus welding is not possible.

K-TIG mitigates the traditionally insufficient energy densities of TIG based techniques by surrounding the tungsten electrode with a cooling shoulder which passes water around it to restrict the high temperature area of the electrode to the tungsten tip. Combined with the magnetic 'pinching' effect in plasmas - a process whereby the flow of charged particles induce a magnetic field around themselves which in turn constricts the flow of the charged particles - the arc jet diameter is restricted and thus the energy density of the arc in increased [18]. When the Arc Pressure reaches a sufficient level, the surface tension of the weld pool can be overcome, and a keyhole is formed [19].

Activated Flux TIG welding was invented in Ukraine in 1960 [20]. It involves the addition of surfactants to the surface of the work piece so that the Marangoni flow is radially inwards. Typically, the hotter regions in a molten metal will have a lower surface tension than the cooler regions however, with the addition of a flux containing Chalcogens such as Oxygen, Sulfur and Selenium this flow can be reversed. This is due to the increase in surface tension with temperature they cause within the weld pool. Typically, surface tension decreases with temperature but through the addition of Chalcogens this is reversed and as temperature drops off at $500 \mathrm{Kmm}^{-1}$ from the centre of the weld pool there is a higher surface tension at the centre of the weld pool. This is illustrated in Figure 1, showing ideal Marangoni convection for deep penetration welding where surface tension is marked $\mathrm{Y}$. This effect is particularly important when welding steel as it has been found that if the concentration of Sulphur or oxygen exceeds $50 \mathrm{ppm}$ the Marangoni flow will be radially inwards [21] - the ideal situation for deep penetration welding.

\subsection{The 'Red Region' of current}

Using a thermal camera, the Red Region of current was first observed at The University of Sheffield; at welding currents above 300A, significant surface instability began with vigorous fluctuations observed. Oscillating fluctuations of the weld pool surface is indicative of competing forces. Typically, arc pressure pushes the surface of the weld pool downwards and in K-TIG this effect is used to form the keyhole.

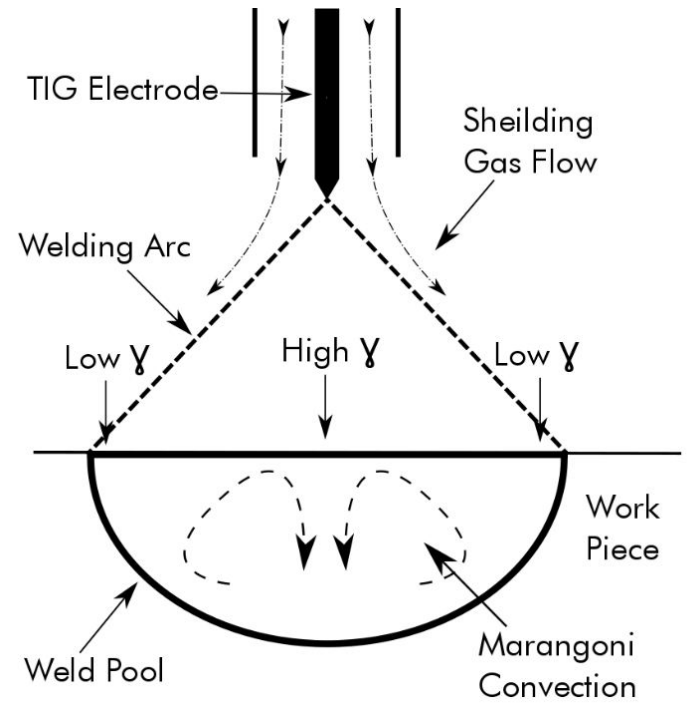

Fig. 1. Ideal Marangoni convection for deep penetration welding.

Conversely, a characteristic of inwards Marangoni convection is a raising of the weld pools surface, thus there are documented effects which raise and lower the surface of the weld pool. However, this is not a sufficient explanation for the fluctuations observed as the Marangoni convection depends on the temperature dependence of the surface tension of the work piece, meaning that temperature would decrease as the arc pressure pushes the surface of the weld pool away from the maximum in the arc temperature near the electrode tip [22]. This effect was first observed when welding with a VBCie 500DHC so to confirm the fluctuations in the weld pool are not an artefact of the VBCie 500DHC, the Miller Dynasty 350 is also used in this study. There is not an obvious explanation as to the origin of the 'Red Region' and as to why specifically 300 A triggers such vigorous effects. By understanding the 'Red Region' it could potentially be manipulated for deep penetration welding. The following experimental procedure will explain the firsts attempts to understand and quantify the its effects by investigating the Voltage, Current and Power used by the two aforementioned welding machines and their performance at high amperages.

\subsection{Industry-Academia Collaboration}

Since 2006, The University of Sheffield has been involved in an industry-academia collaboration with VBC Instrument Engineering. The chief focus of the collaboration has been knowledge transfer of technologies developed at Sheffield for the ATLAS detector at the CERN Large Hadron Collider [23]. Specifically, the low heat input welding technology developed for the construction of the titanium alloy based cooling system for the forthcoming upgraded ATLAS detector has been integrated into commercial welding machines produced by VBC Instrument Engineering. 


\section{Experimental Procedure}

The experiment involved comparing the efficacy of the welds produced by the Miller Dynasty 350 (Miller), the VBCie 500DHC without the DHC feature but with the InterPulse feature (Interpulse) [24-25] and the VBCie with the DHC and InterPulse features (DHC). Weld efficacy was accessed through investigating electrical measurements for the current produced by the machines compared to the current level the machines were set to. These electrical measurements were taken with a custom built shunt box [26]. In addition to this, the power consumption of the machines was investigated by measuring the power drawn from the mains.

The welding was performed on pieces of 22 inch OD, 0.5 inch wall thickness, 316 stainless steel tubing. The tubing was manipulated using a VBC Model W Assisted Hinge turntable. The welding torch was precisely positioned above the stainless steel tubing using a set of linear positioners to adjust the height and keep the arc gap at $3 \mathrm{~mm}$ for all of the machines. The experimental set-up is illustrated in Figure 2.

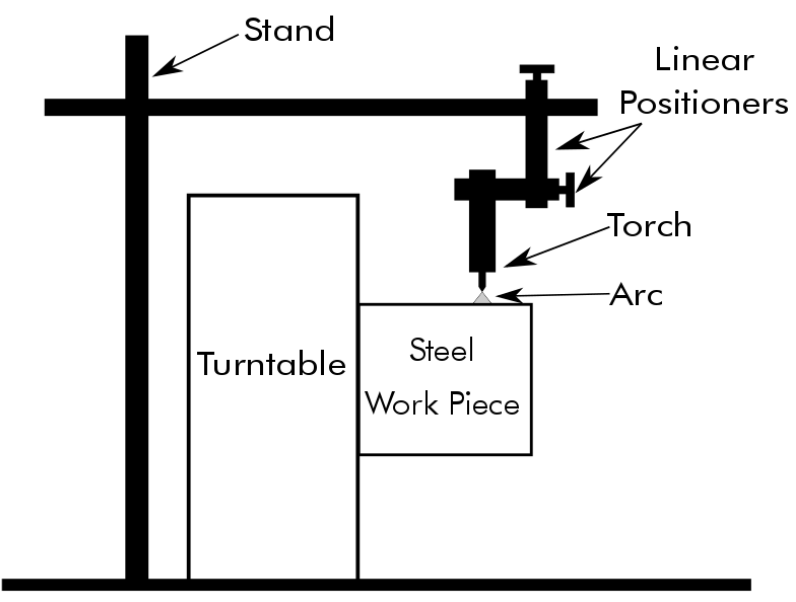

Fig. 2. Schematic of the experimental set-up used to perform the welding. Both the turntable and torch were connected to the welding machine to synchronise the welding process.

Welds were produced at 100A, 150A and 200A for all three machines. These values were chosen to explore a broad range of higher current values. The current measurements were taken using a custom built shunt box which sampled the measurements as an analogue signal. To eliminate noise, a $5 \mathrm{~Hz}$ lowpass filter was applied to the data. This frequency was found to best isolate the signal from the background. The turntable controlled the welding speed and distance so that each weld was $5.76 \mathrm{~cm}$ long and welded at a speed of $35.9 \mathrm{~cm}$ per minute.

\subsection{DHC}

The VBCie 500DHC features a Dynamic Heat Control (DHC) mode which is designed to facilitate superior weld quality. The DHC mode alters the welding current profile to manipulate the heat input and improve weld quality. This is achieved by gradually reducing the current once the weld pool has been formed and thus mediating the heat input to the workpiece. This current drop can be tuned specifically to the job requirements.

\section{Results and discussion}

The current, voltage and power drawn from the mains were each measured three times for each machine at each current setting. The current levels on each of the welding machines were set to $100 \mathrm{~A}, 150 \mathrm{~A}$ and 200A. The average values of the measurements for each setting are shown in Table 1. The current profiles at 150A for each machine is shown in Figure 3.

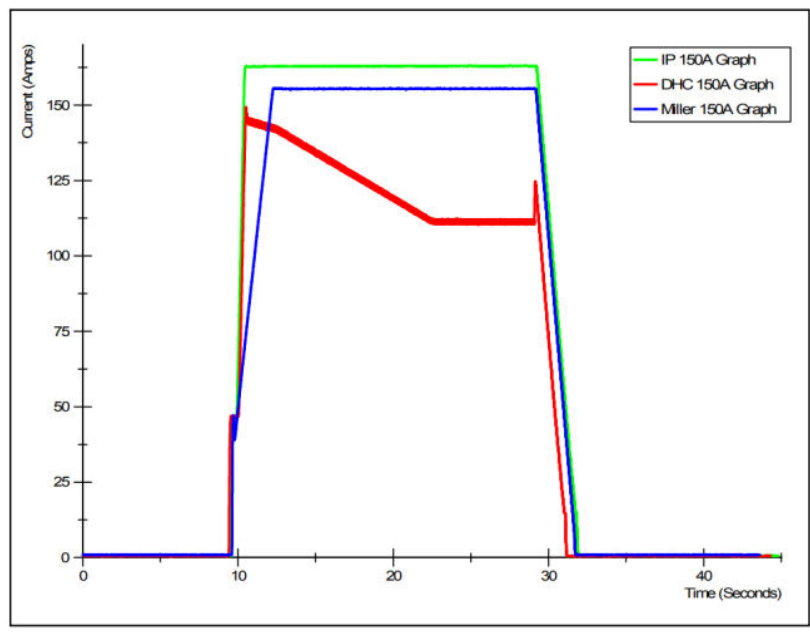

Fig. 3. Current profile for each machine at $150 \mathrm{~A}$. The current profile from the Miller machine (Miller) is shown in blue, the profile of the VBC machine without DHC (IP) is shown in green and the profile of the VBC machine with DHC (DHC) is shown in red.

Table 1. Average of data collected for each machine at each setting.

\begin{tabular}{|l|c|c|c|}
\hline \multicolumn{1}{|c|}{ Machine } & $\begin{array}{c}\text { Measured } \\
\text { Current } \\
\text { (A) }\end{array}$ & $\begin{array}{c} \pm \% \\
\text { Deviation }\end{array}$ & $\begin{array}{c}\text { Power from } \\
\text { Mains } \\
\text { (Watts) }\end{array}$ \\
\hline Miller 100A & 104.12 & +4.12 & 2976.92 \\
\hline Miller 150A & 155.07 & +3.38 & 3753.64 \\
\hline Miller 200A & 205.66 & +2.83 & 4909.96 \\
\hline $\begin{array}{l}\text { InterPulse } \\
\text { 100A }\end{array}$ & 111.7 & +11.7 & 3051.14 \\
\hline $\begin{array}{l}\text { InterPulse } \\
\text { 150A }\end{array}$ & 162.84 & +8.56 & 4481.83 \\
\hline $\begin{array}{l}\text { InterPulse } \\
\text { 200A }\end{array}$ & 214.2 & +7.1 & 6169.27 \\
\hline DHC 100A & 84.92 & +6.33 & 2431.96 \\
\hline DHC 150A & 122.22 & +2.02 & 3354.37 \\
\hline DHC 200A & 160.03 & +0.19 & 4491.21 \\
\hline
\end{tabular}


The current profiles were compared to the values of current that the machines were set to and the percentage deviation from the set value could then be determined. For instance, the average current level from the profiles in Figure 3 would be compared to 150A. In determining the accuracy of the DHC between its stated and delivered current, its unique current profile was accounted for.

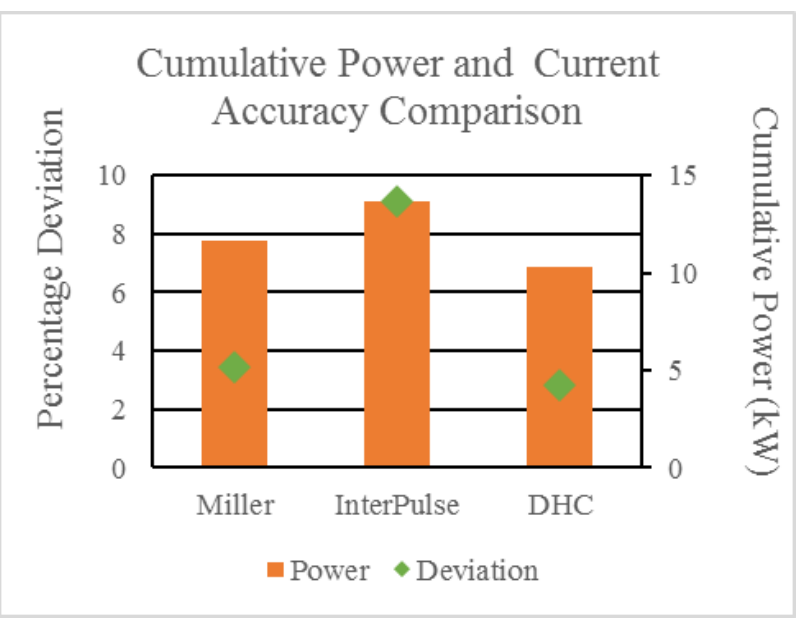

Fig. 4. Cumulative Power and Current Accuracy Comparison

Figure 4 shows a comparison of the average percentage deviation over the three welding currents for each machine. It also shows the cumulative power used by each machine; this was calculated by finding the average power at each current level and then summing these values for each machine. The VBCie 500DHC with the DHC mode on boasts both the lowest cumulative power consumption and the lowest percentage deviation - the highest accuracy - of all the configurations tested.

\section{Conclusion}

This comparison suggests both machines are capable of reasonable accuracy at higher currents. Furthermore, the current profiles on both machines can be identified and processed and thus any interesting activity in the 'Red Region' can be recognized.

This preliminary investigation into the 'Red Region' lays the ground for future work. The next step would be to establish a computational model which reflects the findings of this paper and a welding vision system. In addition to this, voltage measurements could be taken so that the power generated by the machines can be found and thus, the efficiency of the machines can be calculated. A welding vision system is currently under development at The University of Sheffield as is the development of a voltage sensor for high welding currents.

\section{References}

1. Singh, V. Dey, R. Rai, $5^{\text {th }}$ International Conference of Materials Processing and Characterization (ICMPC 2016), Proceedings 4, 1252-1259, (2017)
2. Shirvan, I. Choquet, Weld World, 60:821-835, (2016)

3. M. Lin, T Eagar, $65^{\text {th }}$ Annual AWS Convention, Texas, April 8-13, (1984)

4. Y. Feng, Z. Luo, Z. Liu, Y. Li, y. Luo, Y. Huang, Materials and Design 85, 24-31, (2015)

5. S. Han et al, Weld World, 57:257-264, (2013)

6. V. Nemchinsky, J. Phys. D: Appl. Phys. 29 2659, (1996)

7. J. Wang et al, Int. J. Adv. Manuf. Technol., 90:413420, (2017)

8. H. Ham, D. Oh, S. Cho, Science and Technology of Welding and Joining, 17:7, 594-600, (2012)

9. P. Kah et al, International Journal of Mechanical and Materials Engineering, 9:15, (2014)

10. A. Shirvan, I. Choquet, H. Nilsson, J. Phys. D: Appl. Phys. 49 485201, (2016)

11. A. Faraji et al, Int. J. Adv. Manuf. Technol., 71:2059-2071, (2014)

12. A. Murphy et al, International Conference on CFD in the Minerals and Process Industries CSIRO, Melbourne, Australia 9-11 December 2009, (2009)

13. M. Tanaka et al, Metallurgical and Materials Transactions A, Volume 33A, July 2002-2043, (2002)

14. I. Choquet et al, Proceedings of the Swedish Production Symposium, SPS11, May 3-5, Lund, Sweden, pp. 202-211,( 2011)

15. B. Jarvis, $\mathrm{PhD}$ thesis, University of Wollongong, Wollongong, New South Wales, Australia, (2001)

16. T. Eagar, Advances in Welding Science \& Technology, (1986)

17. J. Lancaster, Physics in Technology 15 73, (1984)

18. Z. Liu et al, Journal of Materials Processing Technology 238 65-72, (2016)

19. W. Fan et al, Int J Adv Manuf Technol 92:22072216, (2017)

20. H. Fujii, Welding International, 19:12, 934-939, (2005)

21. K. Mills et al, Phil. Trans. R. Soc. Lond. A, 356: 911-925. (1998)

22. G. Haddad, Welding J. 64, 339s, (1985)

23. The ATLAS Collaboration, Journal of Instrumentation, Volume 3, (2008)

24. R. Leary et al, Materials Science and Engineering A, 527, 7694-7705, (2010)

25. R. French, H. Marin-Reyes, IIW International Conference, 2 - 3 July, Helsinki, Finland, (2015)

26. R. French, H. Marin-Reyes, M. Benakis, $8^{\text {th }}$ International Conference on Applied Human Factors and Ergonomics (AHFE 2017), 17-21 Jul 2017, Los Angeles, California, USA, (2017) 